\title{
IMPACTO VOLUMÉTRICO E ECONÔMICO PARA DIFERENTES SORTIMENTOS EM TORAS DE PINUS
}

\author{
VOLUME AND ECONOMIC IMPACT FOR DIFFERENT ASSORTMENTS IN PINUS LOGS
}

\author{
Edson Luis Serpe', Afonso Figueiredo Filho ${ }^{2}$, Julio Eduardo Arce \\ ${ }^{1}$ LWARCEL, Lençóis Paulista, São Paulo, Brasil - serpe.edson@gmail.com \\ ${ }^{2}$ Universidade Estadual do Centro Oeste, Irati, Paraná, Brasil-afigfilho@gmail.com \\ ${ }^{3}$ Universidade Federal do Paraná, Curitiba, Paraná, Brasil - jarce@ufpr.br
}

RESUMO

\begin{abstract}
O objetivo deste trabalho é testar diferentes alternativas de sortimentos para o censo florestal, avaliando o retorno econômico das melhores alternativas, comparando-as com o sortimento padrão da empresa. O impacto volumétrico e econômico para diferentes sortimentos em toras de pinus, foram estudados no município de Bituruna, região sul do estado do Paraná. Apesar de ser possível matematicamente a realização de inúmeros sortimentos que poderiam proporcionar melhores rentabilidades, o polo regional consumidor de madeira direciona a especificação dos tipos de produtos a serem produzidos pela floresta. As estimativas do volume total e comercial (diâmetro mínimo até $8 \mathrm{~cm}$ com casca), sortimentos e respectivas simulações, foram realizadas no software Fsign. Os resultados obtidos demonstraram que o sortimento utilizado influenciou diretamente no volume comercial, variando entre $-21,51$ a $1,94 \%$ e a receita bruta impactada negativamente em até $-19,1 \%$.
\end{abstract}

PALAVRAS-CHAVE: Colheita florestal; Perdas; Rentabilidade; Simulação.

\section{ABSTRACT}

The objective of this work is to test different alternatives of assortments for the forest census, evaluating the economic return of the best alternatives, comparing them with the standard assortment of the company. The volumetric and economic impact for different assortments in pine logs were studied in the municipality of Bituruna, southern region of Paraná State. Although it is possible mathematically to make numerous assortments that could provide better returns, the regional pole consuming timber directs the specification of the types of products to be produced by the forest. Estimates of total and commercial volume (minimum diameter up to $8 \mathrm{~cm}$ in shell), assortments and respective simulations were performed in Fsign software. The results showed that the assortment used influenced directly the commercial volume, ranging from -21.51 to $1.94 \%$ and the gross revenue negatively impacted up to $-19.1 \%$.

KEY WORDS: Forest harvest; Losses; Profitability; Simulation. 


\section{INTRODUÇÃO}

A madeira de Pinus devido sua versatilidade está presente nas cadeias produtivas de diferentes produtos, podendo ser destinada a diferentes indústrias, para os mais diversos fins, sendo utilizada para celulose fibra longa não branqueada, pasta mecânica, molduras, painéis de madeira reconstituída (compensado, MDF, MDP, OSB e SDF), madeira serrada, móveis, palito de fósforo e papel e celulose (SCHUCHOVSKI, 2003; BOGNOLA et al., 2008; AGUIAR et al., 2011).

O sortimento florestal é o termo utilizado para resumir a avaliação comercial quali-quantitativa da floresta em pé a partir de uma lista de diferentes multiprodutos (ARCE, 2000). O sortimento é o "seccionamento" da árvore pelas dimensões diâmetro e comprimento (SILVA \& SILVA, 1982).

A pesquisa do sortimento dos plantios é importante para o planejamento do manejo florestal. O sortimento das árvores permite a obtenção de multiprodutos e esses agregam valor à madeira (ARCE, 2000).

O corte raso é a operação de uma rotação de produção florestal, na qual são obtidos os produtos mais valiosos, constituindo um dos fatores que determinam a viabilidade florestal. A maneira como é conduzido o processamento dos fustes é fundamental para estabelecer as quantidades de cada um dos diferentes tipos de produtos que podem ser obtidos da floresta (ARCE et al., 2004).

A utilização de um único comprimento de tora torna a tomada de decisão bem mais simples (pulpwood), porém no caso de multiprodutos florestais (utility e clearwood) a complexidade combinatória necessária para atingir o traçamento otimizado aumenta (ARCE et al., 2004).

Muitas empresas utilizam, comumente, uma variedade de comprimentos de toras de acordo com os seus objetivos comerciais, mas procurando sempre a maximização dos lucros. Com essa intenção, apostam em comprimentos alternativos de toras, visando um melhor aproveitamento do material lenhoso (CAMPOS \& TAVARES, 2010).

$O$ objetivo deste trabalho é testar diferentes alternativas de sortimentos para o censo florestal em um povoamento de Pinus, avaliando o retorno econômico das melhores alternativas, comparando-as com o sortimento padrão da empresa.

\section{MATERIAL E MÉTODOS}

\section{Localização da área de estudo}

A área de estudo localiza-se no município de Bituruna, estado do Paraná, nas coordenadas UTM (Universal
Transversa de Mercator) 431.383,731 m E e 7.104.262,170 m N (Datum Sirgas 2000, Zona 22J), em uma altitude média de 1.216 m.s.n.m. Predominam solos denominados neossolos litólicos, cambissolos e argissolos.

\section{Coleta dos dados}

Os dados foram coletados em uma área de 43,6 ha de plantio misto de Pinus elliottii Engelm e Pinus taeda L. implantado em relevo suave ondulado (declividade média 7\%). A implantação deste povoamento foi realizada no ano de 1998, com espaçamento de $3 \mathrm{~m} \times 3 \mathrm{~m}$.

O mesmo foi conduzido com manejo clearwood, sendo realizado o primeiro desbaste seletivo baixo (colheita de árvores suprimidas ou com problemas de qualidade como: tortuosidades, bifurcadas ou com danos causados pelo ataque de pragas).

O primeiro desbaste foi realizado aos 9 anos de idade, remanescendo 667 árvores.ha-1, o segundo desbaste seletivo baixo aos 12 anos de idade, remanescendo 440 árvores.ha-1 e o corte raso aos 16 anos de idade. Além disso, foram realizadas podas até $6 \mathrm{~m}$ de altura.

Nesta pesquisa, foi realizado o inventário a $100 \%$ (censo florestal), quando o plantio tinha 16 anos de idade, sendo mensurados o diâmetro a 1,3 m do solo (DAP), com a utilização de uma suta, e a altura total mensuradas com o hipsômetro Vertex, de todas as 19.329 árvores.

\section{Estimativa do volume e sortimentos}

O volume comercial (diâmetro mínimo de $8 \mathrm{~cm}$ com casca) e os sortimentos do censo foram estimados com a integral do polinômio de potências, ajustado por Kohler (2017), com árvores cubadas na área de estudo.

$$
\begin{gathered}
\frac{\mathrm{di}}{\mathrm{DAP}=1,4028}+0,4226\left(\frac{\mathrm{hi}}{\mathrm{h}}\right)^{0,005}-0,2691\left(\frac{\mathrm{hi}}{\mathrm{h}}\right)^{0,7} \\
-1,0098\left(\frac{\mathrm{hi}}{\mathrm{h}}\right)^{0,1}-4,2462\left(\frac{\mathrm{hi}}{\mathrm{h}}\right)^{6} \\
+3,8063\left(\frac{\mathrm{hi}}{\mathrm{h}}\right)^{7} \\
\mathrm{R}^{2}{ }_{\mathrm{aj}}=0,974 \quad \mathrm{~S}_{\mathrm{yx}}=9,6 \%
\end{gathered}
$$

Em que: di = diâmetro $(\mathrm{cm})$ com casca na altura hi $(\mathrm{m})$; DAP = diâmetro a 1,3 $\mathrm{m}$ do solo; hi = altura $(\mathrm{m})$ até um diâmetro di $(\mathrm{cm}) ; \mathrm{h}=$ altura $(\mathrm{m}) ; \mathrm{R}^{2}{ }_{\mathrm{aj}}=$ coeficiente de determinação ajustado; $\mathrm{S}_{\mathrm{yx}}=$ erro padrão de estimativa em $\%$.

Os sortimentos foram definidos, de acordo com os praticados pela empresa e também para os praticados pelo 
mercado regional. As estimativas do volume total e comercial (diâmetro mínimo até $8 \mathrm{~cm}$ com casca), sortimentos e respectivas simulações descritas no próximo item, foram realizadas no software Fsign, desenvolvido e autorizado para esta pesquisa pela Brisa Consulting.

\section{Simulação de diferentes grupos de sortimentos de toras}

Apesar de ser possível matematicamente a realização de inúmeros sortimentos que poderiam proporcionar melhores rentabilidades, o polo regional consumidor direciona os tipos de produtos a serem produzidos.

Os trabalhos de Eisfeld et al. (2004), Menon (2005), Lazaretti (2007), Oliveira \& Lopes (2009), Sponholz (2012), Rosa \& Oliveira (2014) e Drinko et al. (2015) relataram os sortimentos utilizados em polos regionais consumidores de multiprodutos do Paraná e Santa Catarina.

Além dos sortimentos utilizados nos polos regionais citados, a Secretaria da Agricultura e Abastecimento do Paraná (SEAB), publica semestralmente o levantamento de preços de produtos florestais com os sortimentos praticados pelos diversos polos regionais consumidores de madeira do estado do Paraná.

Assim, diferentes grupos de sortimentos de toras para a floresta foram estimados com dados do censo florestal, a fim de comparar as classes de sortimentos praticadas pela empresa em estudo (testemunha) e os sortimentos praticados pelo mercado de toras na região sul, sendo cadastrados e simulados 10 grupos de sortimentos no software Fsign (Tabela 1).

Os sortimentos foram calculados para cada árvore mensurada no censo florestal e em seguida extrapolado para toda a área e posteriormente dividido pelo número de hectares.

\section{Análise econômica para diferentes grupos de sortimentos}

Para a simulação da maximização da receita bruta de diferentes grupos de sortimentos, foi utilizado os dados do censo florestal.

Foi calculado o volume comercial por grupo de sortimentos utilizando o padrão da empresa em estudo e o volume por grupo de sortimentos para os sortimentos praticados pelo mercado regional de madeira (Tabela 1), sendo esses comparados.

A avaliação econômica considerou os preços de madeira praticados pela empresa na região de União da Vitória, estado do Paraná (Tabela 2).
Tabela 2. Preços de madeira praticados na região de União da Vitória, estado do Paraná

\begin{tabular}{ccc}
\hline Classe & Classe de diâmetro $(\mathbf{c m})$ & $\mathbf{R} \mathbf{\$} / \mathbf{m}^{\mathbf{3}}$ \\
\hline Serraria 1* & $\geq 23$ & 122,40 \\
\hline Serraria 1** & $\geq 25$ & 127,42 \\
Serraria 2* & $\geq 16$ & 73,69 \\
Serraria 2** & $\geq 18<23$ & 79,78 \\
Serraria 2** & $\geq 18<25$ & 85,87 \\
Celulose* & $\geq 8$ & 32,53 \\
Celulose** & $\geq 8$ & 37,68 \\
\hline *: Sortimentos utilizados pela empresa; **: & Sortimentos \\
considerados pelo mercado regional. &
\end{tabular}

\section{RESULTADOS E DISCUSSÃO}

\section{Simulação para diferentes grupos de sortimentos}

A simulação do volume comercial e variação percentual nos diferentes grupos de sortimentos são apresentados na Tabela 3. Os resultados do volume comercial por hectare, foram relacionados diretamente aos diferentes sortimentos simulados, ou seja, o volume variou dependendo do sortimento utilizado, em que a variação em relação à testemunha foi de $-21,51$ a 1,94\%.

0 volume comercial da simulação 7, quando comparado à testemunha, considerando as classes de sortimentos utilizadas pela empresa, identificou um percentual inferior em $-0,71 \%$, sendo a menor variação encontrada.

Silva et al. (2015), em pesquisa de calibração de uma heurística de construção por partes para otimizar o sortimento florestal em plantios de $P$. taeda, verificaram que, em função dos diferentes sortimentos, o volume comercial foi subestimado entre 0,06 e $0,39 \%$.

A pesquisa de otimização de multiprodutos em plantios de Eucalyptus grandis Hill ex Maiden, considerando produtos individualizados (celulose, energia e serraria) com diferentes sortimentos, apresentou o volume comercial inferior, variando em celulose (6,85\%), energia $(0,55 \%)$ e serraria $(9,82 \%)$ quando comparado à utilização da combinação dos produtos individualizados e multiprodutos (SOARES et al., 2003).

Campos \& Tavares (2010), em pesquisa da influência do comprimento e otimização de toras em Pinus pinaster Aiton, verificaram que o volume colhido foi inferior em $3,81 \%$ ao volume simulado na otimização.

Comparando a pesquisa atual com Soares et al. (2003), Campos \& Tavares (2010) e Silva et al. (2015), observou-se que, nessas pesquisas, a utilização de diferentes sortimentos refletiu diretamente no volume, com tendência de subestimação. 
Tabela 1. Grupos de sortimentos de toras simulados

\begin{tabular}{|c|c|c|c|c|c|}
\hline Classe & Serraria 1 & Serraria 1 & Serraria 2 & Serraria 2 & Celulose \\
\hline Diâmetro (cm) & $\geq 25$ & $\geq 23$ & $\geq 18$ & $\geq 16$ & $\geq 8$ \\
\hline Testemunha* & - & $2,65^{* *}$ & - & 2,65 & 2,3 \\
\hline 1 & - & 3,05 & - & 2,65 & 2,3 \\
\hline 2 & - & 2,65 & 1,9 & - & 2,4 \\
\hline 3 & - & 2,65 & 2,35 & - & 2,4 \\
\hline 4 & 2,35 & - & 1,9 & - & 2,4 \\
\hline 5 & 3,05 & - & 1,9 & - & 2,4 \\
\hline 6 & 2,35 & - & 2,35 & - & 2,4 \\
\hline 7 & 2,65 & - & 2,65 & - & 2,4 \\
\hline 8 & 3,05 & - & 2,65 & - & 2,4 \\
\hline 9 & 3,05 & - & 2,65 & - & 2,6 \\
\hline
\end{tabular}

*: Sortimentos produzidos pela empresa na área de estudo; **: Comprimento da tora $(\mathrm{m})$.

Tabela 3. Volume comercial, variação percentual por grupos de sortimentos e volume por sortimentos

\begin{tabular}{cccccc}
\hline \multirow{2}{*}{ Simulação } & Volume Comercial $\left(\mathbf{m}^{\mathbf{3}} \cdot \mathbf{h a}^{-\mathbf{1}}\right)$ & Variação* (\%) & \multicolumn{3}{c}{ Sortimento $\left(\mathbf{m}^{\mathbf{3}} \cdot \mathbf{h a}^{-\mathbf{1}}\right)$} \\
\cline { 4 - 6 } & & & Serraria 1 & Serraria 2 & Celulose \\
\hline $\mathbf{1}$ & 227,17 & $-6,98$ & 138,41 & 69,06 & 19,70 \\
$\mathbf{3}$ & 215,54 & $-11,74$ & 142,90 & 44,16 & 28,47 \\
$\mathbf{4}$ & 235,63 & $-3,52$ & 142,89 & 55,94 & 36,81 \\
$\mathbf{5}$ & 221,16 & $-9,44$ & 111,58 & 77,05 & 32,53 \\
$\mathbf{6}$ & 191,68 & $-21,51$ & 103,43 & 61,26 & 26,99 \\
$\mathbf{7}$ & 248,97 & 1,94 & 111,59 & 96,38 & 41,01 \\
$\mathbf{8}$ & 242,49 & $-0,71$ & 108,17 & 97,58 & 36,74 \\
$\mathbf{9}$ & 223,83 & $-8,35$ & 103,43 & 87,11 & 33,28 \\
\hline Testemunha & 246,38 & 0,88 & 108,19 & 97,57 & 40,63 \\
\hline
\end{tabular}

*: Variação em relação a testemunha (\%).

\section{Receita bruta para diferentes grupos de sortimentos}

A determinação da receita bruta levou em consideração o volume estimado nas diferentes simulações e o volume por sortimento.

$\mathrm{Na}$ Figura 1 são apresentadas as receitas brutas por hectare para os diferentes grupos de sortimentos.

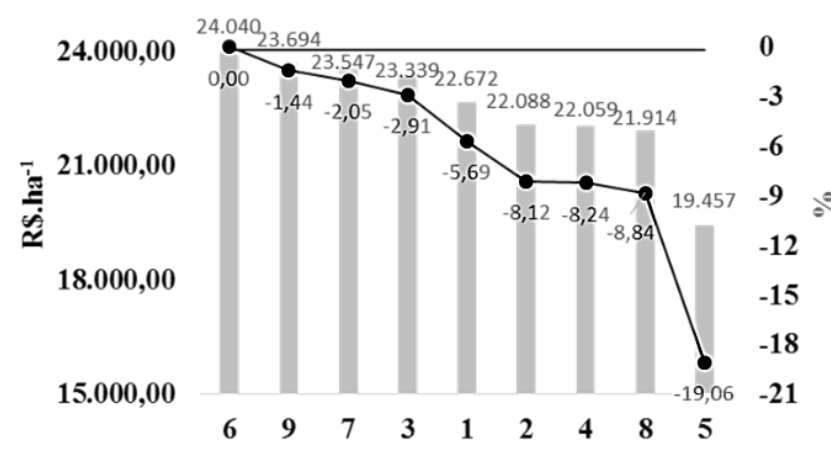

Simulação — Testemunha $\rightarrow$-Variação em relação à testemunha

Figura 1. Receita bruta para diferentes grupos de sortimentos.

Verificou-se que o grupo de sortimento 6 apresentou a maior receita bruta, por apresentar maior volume comercial e adequada otimização para o fuste nas classes de sortimento.

Ao serem definidos os grupos de sortimentos, a receita bruta pode ser alterada drasticamente e o impacto negativo pode variar de 0 a 19,1\%.

Os grupos de sortimentos simulados apresentaram a tendência de subestimar a receita bruta quando comparado com a testemunha.

Silva et al. (2015) constataram que a calibração de uma heurística de construção por partes para otimizar o sortimento florestal em plantios de $P$. taeda, em função dos diferentes sortimentos, a receita bruta é impactada negativamente entre 0,45 e $1,28 \%$, dependendo do sortimento definido para o processamento de fuste.

Menon (2005), em pesquisa de meta-heurísticas na otimização do sortimento florestal de $P$. taeda, observou que a receita bruta, quando comparada com a dos sortimentos utilizados pela empresa com os mesmos sortimentos utilizando a meta-heurística, os cenários otimizados a subestimaram entre 7,48 e 9,55\%.

Em proposta de otimização de eucalipto utilizando multiprodutos, constatou-se que ao comparar o cenário ótimo de sortimento a diferentes opções de classes de 
sortimentos, a receita bruta é alterada podendo variar negativamente entre 0,90 e 12,50\% (SARTORI, 2013).

Comparando os resultados obtidos por Menon (2005) e Sartori (2013) a esta pesquisa, verificou-se que o uso de diferentes grupos de sortimentos pode subestimar a receita bruta.

\section{CONCLUSÕES}

As classes de sortimento utilizadas para a estimativa de volume comercial impactaram em maior ou menor volume, proporcionando distintos resultados na receita bruta.

As classes de sortimento de toras utilizadas pela empresa proporcionaram resultados econômicos lucrativos, ao comparar com os sortimentos simulados.

A definição das classes de sortimentos de madeira para a comercialização no mercado, necessitam passar pelo planejamento florestal de maneira a realizar simulações volumétricas e análise da receita gerada, buscando maximizar os resultados.

\section{AGRADECIMENTOS}

À Remasa Reflorestadora S.A., pela autorização e apoio no trabalho de campo. A Brisa Consulting pela disponibilização do software Fsign.

\section{REFERÊNCIAS}

AGUIAR, A.V. et al. Programa de melhoramento de pinus da Embrapa Florestas. Documentos, n.233, p.1-81, 2011.

ARCE, J.E. Um sistema de análise, simulação e otimização do sortimento florestal em função da demanda por multiprodutos e dos custos de transporte. 2000. 125p. (Tese de doutorado).

ARCE, J.E. et al. Geração de padrões ótimos de corte através de algoritmos de traçamento aplicados a fustes individuais. Árvore, v.28, n.2, p.207-217, 2004.

BOGNOLA, I.A. et al. Modelagem uni e bivariada da variabilidade espacial de rendimento de Pinus taeda L. Floresta, v.38, n.2, p.373-385, 2008.

CAMPOS, C.; TAVARES, M. Influência do comprimento e origem do toro na optimização do aproveitamento e do valor de rolos de pinho bravo. Silva Lusitana, v.18, n.1, p.123-132, 2010.

DRINKO, C.H. et al. Produtividade e custos do corte de pinus com harvester de pneus e esteiras. Enciclopédia Biosfera, v.11, n.22, p.3664-3677, 2015.

EISFELD, R.L. et al. Avaliação de modelos polinomiais na estimativa de volume total e por sortimento de Pinus taeda. Brasil Florestal, v.23, n.79, p.9-15, 2004.
KOHLER, S.V. Erros amostrais e não amostrais em inventários de florestas plantadas. 2017. 173p. (Tese de doutorado).

LAZARETTI, D.S. Qualidade da madeira e rentabilidade na produção de Pinus taeda L. visando múltiplos mercados. 2007. 130p. (Dissertação de mestrado).

MENON, M.U. Meta-heurísticas na otimização do sortimento florestal. 2005. 119p. (Tese de doutorado).

OLIVEIRA, D.; LOPES, E. S. Análise da influência das dimensões das toras de pinus na produtividade e custo da extração florestal. XVIII Encontro Anual de Iniciação Científica, p.4-14, 2009.

ROSA, M.O.; OLIVEIRA, F.M. Análise da qualidade do processamento de madeira em dois sistemas mecanizados de colheita florestal. Revista da União Latino-americana de Tecnologia, n.2, p.19-37, 2014.

SARTORI, M.S. Proposta de otimização para reflorestamento de eucalipto utilizando multiprodutos. 2013. 70p. (Tese de doutorado).

SCHUCHOVSKI, M.S. Diagnóstico e planejamento do consumo de madeira e da produção em plantações florestais no estado do Paraná. 2003. 78p. (Dissertação de mestrado).

SILVA, J.A.A.; SILVA, I.P. Estatística experimental aplicada à ciência florestal. Recife: UFPE, 1982.

SILVA, R.F. et al. Meta-heurística Iterated Local Search aplicada ao particionamento de fustes no agronegócio florestal. $\mathbf{X}$ Congresso Brasileiro de Agroinformática, p.4-14, 2015.

SOARES, T.S. et al. Otimização de multiprodutos em povoamentos florestais. Árvore, n.27, p.811-820, 2003.

SPONHOLZ, I.K. Espacialização da produção Pinus taeda por sortimento de madeira. 2012. 51p. (Dissertação de mestrado). 